\title{
Non-negative Matrix Factorization
}

National Cancer Institute

\section{Source}

National Cancer Institute. Non-negative Matrix Factorization. NCI Thesaurus. Code

C64257.

A pattern recognition alog rithm that identifies patterns that together explain the data as a linear combination of expression signatures. 